\title{
Joule-Lenz Energy of Quantum Electron Transitions Compared with the Electromagnetic Emission of Energy
}

\author{
Stanisław Olszewski \\ Institute of Physical Chemistry, Polish Academy of Sciences Kasprzaka, Warsaw, Poland \\ Email: olsz@ichf.edu.pl
}

Received 4 July 2016; accepted 19 August 2016; published 22 August 2016

Copyright @ 2016 by author and Scientific Research Publishing Inc.

This work is licensed under the Creative Commons Attribution International License (CC BY). http://creativecommons.org/licenses/by/4.0/

c) (i) Open Access

\begin{abstract}
In the first step, the Joule-Lenz dissipation energy specified for the electron transitions between two neighbouring quantum levels in the hydrogen atom has been compared with the electromagnetic energy of emission from a single level. Both the electric and magnetic vectors entering the Pointing vector of the electromagnetic field are referred to the one-electron motion performed along an orbit in the atom. In the next step, a similar comparison of emission rates is performed for the harmonic oscillator. Formally a full agreement of the Joule-Lenz and electromagnetic expressions for the energy emission rates has been attained.
\end{abstract}

\section{Keywords}

Joule-Lenz Energy, Quantum Electron Transitions, Hydrogen Atom, Electromagnetic Energy Emission

\section{Introduction}

Usually any calculation of the emission rate of energy in the atom has as its background a rather complicated statistical-and-probabilistic theory. This situation seems to be not changed much since the very end of the nineteenth and beginning of twenteeth century [1]-[3]. In practice an individual atomic system has been never considered, but instead of it an ensemble of the oscillating atoms known as the black body was examined. Rather automatically the temperature parameter-important for comparing the theoretical results with experiment-has been involved in such many-atomic calculations. Next the probabilistic approach to the emission intensity found its justification, and a rather extended though complicated application, in quantum mechanics [4] [5].

More recently an approach to the treatment of the energy emission in a single atomic object could be based on 
the Joule-Lenz law [6]-[10]. For, when the Bohr theory of the hydrogen atom is taken as an example, any atom has its electron placed on a definite orbit which can be approximated by a circle. Electrically such a circular motion can be represented by a current having a known intensity. For example, for the quantum states $n$ and $n+1$ the current intensity is respectively

$$
i_{n}=\frac{e}{T_{n}} \text { and } \quad i_{n+1}=\frac{e}{T_{n+1}}
$$

where $T_{n}$ and $T_{n+1}$ are the time periods of the electron circulation about the proton nucleus. In the next step, the energy difference between levels $n+1$ and $n$, namely

$$
\Delta E=E_{n+1}-E_{n},
$$

provides us with the electric potential

$$
V=\frac{\Delta E}{e} .
$$

This leads to the electric resistance

$$
R=\frac{V}{i} \approx \frac{V}{i_{n}} \approx \frac{V}{i_{n+1}}
$$

where the approximate relations in (4) hold in virtue of

$$
i \approx i_{n} \approx i_{n+1}
$$

valid for large $n$. The validity of (5) becomes evident if we apply Formula (7) in (1).

For such large $n$ we have [11] the energy change

$$
\Delta E=-\frac{m e^{4}}{2 \hbar^{2}}\left[\frac{1}{(n+1)^{2}}-\frac{1}{n^{2}}\right]=\frac{m e^{4}}{2 \hbar^{2}} \frac{(n+1)^{2}-n^{2}}{(n+1)^{2} n^{2}} \approx \frac{m e^{4}}{\hbar^{2} n^{3}} ;
$$

in the last step of (6) the approximation of large $n$ is considered.

Since [11]

$$
T_{n}=\frac{2 \pi n^{3} \hbar^{3}}{e^{4} m}
$$

we obtain

$$
R=\frac{V}{i_{n}}=\frac{\Delta E}{e i_{n}}=\frac{\Delta E T_{n}}{e^{2}} \approx \frac{m e^{4}}{\hbar^{2} n^{3}} \frac{1}{e^{2}} \frac{2 \pi n^{3} \hbar^{3}}{e^{4} m}=\frac{2 \pi \hbar}{e^{2}}=\frac{h}{e^{2}} ;
$$

this is a constant independent of $n$. The same value of $R$ can be calculated also for other quantum systems than the hydrogen atom, see [6] [7] [10]. A characteristic point is that $R$ is equal to a well-known result of experiments done on the integer quantum Hall effect [12].

The Joule-Lenz law is represented by the well-known relation

$$
\frac{\Delta E}{\Delta t}=R i^{2}
$$

where $\Delta t$ is the time interval necessary to produce the emitted energy $\Delta E$. In fact (9) implies that for $i=i_{n}$ we have

$$
\Delta t=\frac{\Delta E}{R i_{n}^{2}}=\frac{m e^{4}}{\hbar^{2} n^{3}} \frac{e^{2}}{h}\left(\frac{T_{n}}{e}\right)^{2}=\frac{m e^{4}}{\hbar^{2} n^{3}} \frac{e^{2}}{h} \frac{1}{e^{2}}\left(\frac{2 \pi n^{3} \hbar^{3}}{e^{4} m}\right)^{2}=\frac{2 \pi}{m e^{4}} \frac{2 \pi}{h} n^{3} \hbar^{4}=\frac{2 \pi n^{3} \hbar^{3}}{m e^{4}}=T_{n} .
$$

Moreover from (6) and (10) we obtain

$$
\Delta E \Delta t=\frac{m e^{4}}{\hbar^{2} n^{3}} \frac{2 \pi n^{3} \hbar^{3}}{m e^{4}}=2 \pi \hbar=h
$$

or 


$$
\Delta t=\frac{h}{\Delta E} .
$$

Therefore the ratio (9) becomes

$$
\frac{\Delta E}{\Delta t}=\frac{(\Delta E)^{2}}{h}=\left(\frac{m e^{4}}{\hbar^{2} n^{3}}\right)^{2} \frac{1}{h}=\frac{m^{2} e^{8}}{2 \pi n^{6} \hbar^{5}} .
$$

Results similar to (10)-(12) can be obtained also for other quantum systems than the hydrogen atom [6] [7] [10].

The principal aim of the paper is, in the first step, to compare the ratio calculated in (13) with the rate of energy emission obtained in terms of the electromagnetic theory. Next, in order to compare the quantum emission with the classical emission rate, the properties of the harmonic oscillator emission are also studied.

\section{Fields Induced by the Electron Motion in the Hydrogen Atom}

The electric field value $\left|\boldsymbol{E}_{n}\right|$ acting on the electron in the Bohr atom is well known:

$$
|\boldsymbol{E}|=\left|\boldsymbol{E}_{n}\right|=\frac{e}{r_{n}^{2}}=\frac{e^{5} m^{2}}{\hbar^{4} n^{4}} .
$$

The last step in (14) is attained because of the radius of the orbit $n$ which is [11]

$$
r_{n}=\frac{\hbar^{2} n^{2}}{m e^{2}}
$$

A less-known magnetic field omitted in the Bohr atomic model [10] is induced in the hydrogen atom due to the circular electron motion done with the frequency

$$
\Omega_{n}=\frac{2 \pi}{T_{n}} .
$$

Because of the formula (see e.g. [13])

$$
\Omega_{n}=\frac{e H_{n}}{m c},
$$

the identity between (16) and (17) combined with (7) gives

$$
\left|\boldsymbol{H}_{n}\right|=H_{n}=\frac{e^{3} m^{2} c}{\hbar^{3} n^{3}} .
$$

A characteristic point is that when expressions for $E_{n}$ and $H_{n}$ are substituted to the Lorentz force

$$
\boldsymbol{F}_{n}=e \boldsymbol{E}_{n}+\frac{e}{c}\left[\boldsymbol{v}_{n} \times \boldsymbol{H}_{n}\right],
$$

we obtain for the electric component of (19)

$$
e\left|\boldsymbol{E}_{n}\right|=\frac{e^{2}}{r_{n}^{2}}=\frac{e^{2}}{n^{4} \hbar^{4}} m^{2} e^{4}=\frac{m^{2} e^{6}}{n^{4} \hbar^{4}}
$$

and the same value is obtained for the magnetic component of (19)

$$
\frac{e}{c}\left|\boldsymbol{v}_{n} \times \boldsymbol{H}_{n}\right|=\frac{e}{c} v_{n} H_{n}=\frac{e}{c} \frac{e^{2}}{n \hbar} \frac{e^{3} m^{2} c}{n^{3} \hbar^{3}}=\frac{m^{2} e^{6}}{n^{4} \hbar^{4}},
$$

on condition the vector of the electron velocity having the value [11]

$$
v_{n}=\frac{e^{2}}{n \hbar}
$$

is normal to $H_{n}$. 


\section{Field Values Specific for the One-Electron Current Present in the Atom and the Electromagnetic Rate of the Energy Emission}

Our aim is to construct the Poynting vector which provides us with the electromagnetic dispense of energy. The vectors $\boldsymbol{E}$ and $\boldsymbol{H}$ become slightly different than in Section 2 because they refer to the current behaviour of the electron which is circulating along its orbit. With the potential $V$ given in (3) and (6) and equal to

$$
V=\frac{m e^{3}}{\hbar^{2} n^{3}},
$$

the electric field on the orbit having the length

$$
l_{n}=2 \pi r_{n}
$$

attains the value [16]

$$
\left|\boldsymbol{E}_{\text {orbit } n}\right|=\frac{V}{2 \pi r_{n}}=\frac{1}{2 \pi} \frac{m e^{3}}{\hbar^{2} n^{3}} \frac{m e^{2}}{n^{2} \hbar^{2}}=\frac{1}{2 \pi n^{5}} \frac{m^{2} e^{5}}{\hbar^{4}} .
$$

This gives an electric vector directed along the current.

On the other hand the magnetic field directed normally to the current attains the value [14] [16]

$$
\left|\boldsymbol{H}_{\text {orbit } n}\right|=\frac{2 i_{n}}{c r_{e}}=\frac{2 e}{T_{n} c r_{e}}=\frac{2 e}{2 \pi n^{3}} \frac{e^{4} m}{\hbar^{3}} \frac{m c^{2}}{e^{2}}=\frac{e^{3} m^{2} c}{\pi \hbar^{3} n^{3}} .
$$

This field differs from that given in (18) solely by the factor equal to $1 / \pi$.

It should be noted that parameter $r_{e}$ entering (26) is the radius of the circular cross-section area of the orbit assumed to be equal to the cross-section of the electron microparticle considered as a sphere [15] [17]:

$$
r_{e} \cong \frac{e^{2}}{m c^{2}} .
$$
[14]

The value of the Poynting vector emanating the energy from the orbit is calculated according to the formula

$$
\begin{aligned}
\left|\boldsymbol{S}_{n}^{P}\right| & =\frac{c}{4 \pi}\left|\boldsymbol{E}_{\text {orbit } n} \times \boldsymbol{H}_{\text {orbit } n}\right| S_{\text {orbit }}=\frac{c}{4 \pi}\left|\boldsymbol{E}_{\text {orbit } n} \| \boldsymbol{H}_{\text {orbit } n}\right| S_{\text {orbit }} \\
& =\frac{c}{4 \pi} \frac{1}{2 \pi n^{5}} \frac{m^{2} e^{5}}{\hbar^{4}} \frac{1}{\pi} \frac{e^{3} m^{2} c}{\hbar^{3} n^{3}} 2 \pi \frac{n^{2} \hbar^{2}}{m e^{2}} 2 \pi \frac{e^{2}}{m c^{2}}=\frac{m^{2} e^{8}}{2 \pi n^{6} \hbar^{5}}
\end{aligned}
$$

where

$$
S_{\text {orbit }}=2 \pi r_{n} 2 \pi r_{e}=4 \pi^{2} r_{n} r_{e}
$$

is the toroidal surface of the orbit having the length (24) and the length of the cross-section circumference of the orbit is equal to

$$
2 \pi r_{e} .
$$

In effect we obtain from (23), (26) and (28) the result precisely equal to Formula (13) calculated from the Joule-Lenz theory. Since (13) assumed the electron transitions solely between the levels

$$
n+1 \rightarrow n,
$$

the identity between (13) and (28) implies that the limitation to transition (31) applies also to the electromagnetic result calculated in (28).

A problem may arise to what extent the energy rate (13), or (28), can be radiated as an electromagnetic wave. An altenative behaviour is that the energy $\Delta E$ is spent for a mechanical rearrangement of the electron position due to the transition process. An argument for that is the presence of the electric force

$$
e\left|\boldsymbol{E}_{\text {orbit } n}\right|=\frac{1}{2 \pi n^{5}} \frac{m^{2} e^{6}}{\hbar^{4}}
$$


along the orbit. The force (32) multiplied by the orbit length calculated in (24) gives

$$
l_{n} e\left|\boldsymbol{E}_{\text {orbit } n}\right|=2 \pi \frac{n^{2} \hbar^{2}}{m e^{2}} \frac{1}{2 \pi n^{5}} \frac{m^{2} e^{6}}{\hbar^{4}}=\frac{m e^{4}}{\hbar^{2} n^{3}}
$$

which is precisely the energy $\Delta E$ of the electron transition obtained in (6).

\section{Quantum and Classical Emission Rate Calculated for the Harmonic Oscillator}

A natural tendency is to compare the quantum rate of the energy emission with the classical emission rate. To this purpose the one-dimensional harmonic oscillator has been chosen as a suitable object of examination.

The classical energy of the oscillator is

$$
E_{\text {osc }}=\frac{k a^{2}}{2},
$$

$a$ is the oscillator amplitude; $m$ is the oscillator mass which together with the force constant $k$ refers to the circular frequency of the oscillator

$$
\omega=\left(\frac{k}{m}\right)^{1 / 2}=\frac{2 \pi}{T}
$$

$T$ is the oscillation period [18].

The quantum oscillator energy is

$$
E_{n}=\left(n+\frac{1}{2}\right) \hbar \omega \cong n \hbar \omega
$$

(the last step holds for large $n$ ) and the change of energy due to transition between the levels $n+1$ and $n$ is

$$
\Delta E=\hbar \omega .
$$

According to the Joule-Lenz approach to the quanta [6]-[10] the emission rate between the levels $n+1$ and $n$ is

$$
\frac{\Delta E}{\Delta t}=\frac{(\Delta E)^{2}}{h}=\frac{(\hbar \omega)^{2}}{h} .
$$

This gives

$$
\frac{1}{\Delta t}=\frac{\Delta E}{h}=\frac{\hbar \omega}{h}=\frac{h v}{h}=v=\frac{1}{T}
$$

SO

$$
\Delta t=T,
$$

because the reference between $\omega$ and $v$ is

$$
\omega=2 \pi \nu .
$$

The potential $V$ connected with the energy change $\Delta E$ is

$$
V=\frac{\Delta E}{e}=\frac{\hbar \omega}{e} \text {. }
$$

If we note that a maximal distance travelled by the electron oscillator in one direction is

$$
l=2 a,
$$

the electric field connected with the oscillator parallel to its motion is

$$
|\boldsymbol{E}|=\frac{V}{l}=\frac{V}{2 a}=\frac{\hbar \omega}{2 a e} .
$$

The electric current let be considered as remaining approximately constant in course of the oscillation. In this case the magnetic field which is normal to the current [see (26)] is 


$$
|\boldsymbol{H}|=\frac{2 i}{c r_{e}}=\frac{2 e}{T c r_{e}}=\frac{2 e}{T c} \frac{m c^{2}}{e^{2}}=\frac{2 m c}{T e}=\frac{2 m c v}{e},
$$

since the cross-section of the electron current is assumed to be identical with the cross-section area of the electron microparticle, see (27).

The surface area of the sample containing the oscillator is

$$
S=2 \pi r_{e} l=4 \pi r_{e} a=4 \pi \frac{e^{2}}{m c^{2}} a,
$$

on condition the contribution of the end areas of the sample surface equal to

$$
2 \pi r_{e}^{2}=2 \pi\left(\frac{e^{2}}{m c^{2}}\right)^{2} \ll 4 \pi \frac{e^{2}}{m c^{2}} a
$$

has been neglected because (47) is a small number in comparison with $S$ in (46).

In consequence, for the vector $\boldsymbol{H}$ normal to vector $\boldsymbol{E}$ the value of the Poynting vector becomes

$$
\left|\boldsymbol{S}^{P}\right|=\frac{c}{4 \pi}|\boldsymbol{E}||\boldsymbol{H}| S=\frac{c}{4 \pi} \frac{\hbar \omega}{2 a e} \frac{2 m c}{T e} 4 \pi \frac{e^{2}}{m c^{2}} a=\frac{4 \pi \hbar \omega}{4 \pi T}=\frac{\Delta E}{T} .
$$

This is a result identical with (38) on condition Formula (39) is taken into account.

According to the classical electrodynamics [19] the emission rate of energy from a classical oscillator is

$$
\frac{\mathrm{d} E}{\mathrm{~d} t}=\frac{2}{3} \frac{\omega^{4}}{c^{3}}|p|^{2}=\frac{2}{3}\left(\frac{2 \pi}{T}\right)^{4} \frac{e^{2} a^{2}}{c^{3}}
$$

since

$$
p=e a
$$

is the dipole moment of the classical harmonic oscillator. Formula (49) can be compared with the quantum approach to the Joule-Lenz emission rate of energy [see (38)]:

$$
\frac{\Delta E}{\Delta t}=\frac{(\hbar \omega)^{2}}{h}=\left(\frac{h}{T}\right)^{2} \frac{1}{h}=\frac{h}{T^{2}} .
$$

In the case of very small quantum systems the amplitude $a$ in (49) can be close to its minimal length [10]

$$
a \cong \frac{\hbar}{m c}
$$

and the time period $T$ can approach its minimal size [10]

$$
T \cong \frac{\hbar}{m c^{2}} .
$$

The equality required between (49) and (51) leads to the relation

$$
\frac{2}{3}\left(\frac{2 \pi}{T}\right)^{4} \frac{e^{2} a^{2}}{c^{3}}=\frac{h}{T^{2}} .
$$

When $a$ and $T$ are taken respectively from (52) and (53), Formula (54) becomes

$$
\frac{2}{3} \frac{(2 \pi)^{4}}{\left(\frac{\hbar}{m c^{2}}\right)^{2}} \cdot \frac{e^{2}\left(\frac{\hbar}{m c}\right)^{2}}{c^{3}}=\frac{2}{3} \frac{(2 \pi)^{4} e^{2}}{c^{-2} c^{3}}=\frac{2}{3} \frac{(2 \pi)^{4} e^{2}}{c}=h
$$

from which we have the relation

$$
\frac{2}{3}(2 \pi)^{3} \approx 165=\frac{\hbar c}{e^{2}}=\frac{1}{\alpha} .
$$


The result obtained in (56) differs by only 20 percent from the reciprocal value of the atomic constant equal to 137.

\section{Ratio of the Classical and Quantum Emission Rate Defined by the Damping Coefficient of the Classical Radiation}

An attempt of this Section is to demonstrate that the classical emission can be considered as a damped quantum emission rate. The classical damping coefficient of the oscillator is [19]

$$
\gamma=\frac{2}{3} \frac{e^{2}}{m c^{3}} \omega^{2} .
$$

On the other hand, the classical emission rate given in (49) can be modified when the amplitude $a$ entering (49) is expressed in terms of the oscillator energy $E$ [18]:

$$
a^{2}=\frac{2 E}{k}=\frac{2 E}{m \omega^{2}}=\frac{2 n \hbar \omega}{m \omega^{2}}=\frac{2 n \hbar}{m \omega} .
$$

Here, at the end of (58), the energy $E$ is replaced by the approximate quantum formula for the oscillator energy given in (36). In effect the classical emission rate in (49) becomes

$$
\eta^{\text {class }}=\frac{2}{3 c^{3}} \omega^{4} e^{2} a^{2}=\frac{2}{3 c^{3}} \omega^{4} e^{2} \frac{2 n \hbar}{m \omega}=\frac{4 e^{2}}{3 c^{3}} \frac{\omega^{3}}{m} n \hbar .
$$

Another transformation may concern the quantum emission rate in (51):

$$
\eta^{\text {quant }}=\frac{\Delta E}{\Delta t}=\frac{h}{T^{2}}=\left(\frac{2 \pi}{T}\right)^{2} \frac{h}{(2 \pi)^{2}}=\frac{\omega^{2} \hbar}{2 \pi} .
$$

As a result of (59) and (60) we obtain the ratio

$$
\frac{\eta^{\text {class }}}{\eta^{\text {quant }}}=\frac{4}{3} \frac{e^{2}}{c^{3}} \frac{\omega^{3}}{m} n \hbar: \frac{\omega^{2} \hbar}{2 \pi}=\frac{8 \pi}{3} \frac{e^{2}}{c^{3}} \frac{\omega}{m} n=4 \pi \frac{2}{3} \frac{e^{2} \omega^{2}}{c^{3} m} \frac{n}{\omega}=4 \pi \gamma \frac{n}{\omega}=2 n T \gamma
$$

which is proportional to $\gamma$ in (57). A multiple of the oscillation time period $T$ is the proportionality coefficient representing (61) in terms of $\gamma$. Therefore another way to write (61) can be

$$
\eta^{\text {class }}=2 n T \gamma \eta^{\text {quant }} \text {. }
$$

Let us note that $E_{n}$ entering (36) and (58) is proportional to $n$.

It is worth to note that the Einstein coefficient $A_{n}^{n-\alpha}$ of the emission probability can be coupled with $\gamma$ by the relation [20]

$$
A_{n}^{n-\alpha} h v=\gamma f_{n, n-\alpha} h v
$$

so

$$
A_{n}^{n-\alpha}=\gamma f_{n, n-\alpha} .
$$

According to Heisenberg [20] [21] we have

$$
|a(n, n-1)|^{2} v(n, n-1)=\frac{n h}{\pi m \omega_{0}} v(n, n-1)=\frac{h}{8 \pi^{2} m} f_{n, n-1}
$$

where $a$ is the quantum-theoretical amplitude of the expansion of the coordinate $x=x(t)$ of an anharmonic oscillator; $\omega_{0}$ is the circular frequency of the harmonic oscillator.

For small peturbation $\lambda$ of the oscillator we have [21]

$$
\omega=2 \pi v(n, n-1) \approx \omega_{0},
$$

so Formula (65) gives

$$
\frac{n h \omega}{2 \pi^{2} m \omega_{0}} \cong \frac{n h \omega_{0}}{2 \pi^{2} m \omega_{0}}=\frac{n h}{2 \pi^{2} m}=\frac{h}{8 \pi^{2} m} f_{n, n-1}
$$


or

$$
4 n=f_{n, n-1} .
$$

In effect for $\alpha=1$ taken in (63) we obtain from (64) and (68):

$$
A_{n}^{n-1}=\gamma f_{n, n-1}=4 n \gamma .
$$

If $\gamma$ is presented, according to (61), in terms of the ratio of $\eta^{\text {class }}$ and $\eta^{\text {quant }}$, we obtain

$$
A_{n}^{n-1}=\frac{4 n}{2 n T} \frac{\eta^{\text {class }}}{\eta^{\text {quant }}}=\frac{2}{T} \frac{\eta^{\text {class }}}{\eta^{\text {quant }}},
$$

where $T$ is the oscillation time period of the harmonic oscillator.

\section{Reciprocal Value of the Atomic Constant and the Electron Spin}

The reciprocal value $\alpha^{-1}$ of the atomic constant $(\sim 137)$ approached in (56) is important in the treatment of the electron spin [10] [22] [23]. We show below that the magnetic field intensity necessary to produce the electron spin can be obtained approximately as a result of a coupling of $\alpha^{-2}$ with the radius $r_{e}$ of the electron microparticle, see (27).

According to the classical electrodynamics [14] the magnetic field $H$ at a distant $r$ from the center of the linear wire carrying a current $i$ is coupled with $i$ and $r$ by the formula

$$
H=\frac{2 i}{c r} \text {. }
$$

If the current $i$ is flowing on a surface of the conductor which is the electron orbit, we can assume that $r=r_{e}$ which is both the radius of the electron microparticle and cross-section of the orbit. The field $H$ becomes in this case [14]

$$
H=\frac{2 e}{T c r_{e}}=\frac{2 e}{c} \frac{e^{4} m}{2 \pi \hbar^{3}} \frac{m c^{2}}{e^{2}}=\frac{e^{3} m^{2} c}{\pi \hbar^{3}},
$$

where the time period $T$ of the electron circulation along the orbit is taken from Formula (7) for $n=1$ :

$$
T=T_{1}=\frac{2 \pi \hbar^{3}}{e^{4} m}
$$

The essence of the spin effect is that the path of the spinning electron circumvents the electron orbit about

$$
\frac{1}{\alpha^{2}}=\left(\frac{\hbar c}{e^{2}}\right)^{2} \cong 137^{2}
$$

times during the time period $T$ indicated in (73). In classical electrodynamics this means that the magnetic field produced in this way is $\alpha^{-2}$ times stronger than that obtained in (72):

$$
H_{\text {spin }}=\frac{1}{\alpha^{2}} H=\frac{\hbar^{2} c^{2}}{e^{4}} \frac{e^{3} m^{2} c}{\pi \hbar^{3}}=\frac{m^{2} c^{3}}{\pi e \hbar} .
$$

The result in (75) differs solely by the factor of $\frac{1}{\pi}$ from the magnetic field assumed to produce a spinning electron particle in [10] [22] [23]:

$$
H_{\text {spin }}=\frac{m^{2} c^{3}}{e \hbar}
$$

A discrepancy between (75) and (76) can be ascribed to some uncertainty connected with the calculation of the radius $r_{e}$, see [24].

\section{Conclusions}

The aim of the paper was to get more insight into a non-probabilistic description of the transfer of energy 
between two quantum levels. A suitable situation for discussion is the case when the levels are neighbouring in their mutual position of the energy states. Then the energy change ( $\Delta E)$ between the levels, and the time interval $\Delta t$ necessary to attain $\Delta E$, satisfy a very simple formula

$$
\Delta E \Delta t=h
$$

see [6]-[10].

In the paper, Formula (77) finds its counterparts supplied by the electromagnetic theory of emission. Two physical objects, namely the hydrogen atom and electron harmonic oscillator, were studied. The case of the electron oscillator allowed us to perform a more direct comparison of the quantum approach to the emission rate with the classical electromagnetic theory. It occurs that the classical rate is equal to the quantum rate multiplied by the Born damping coefficient and an interval of time, see (62).

\section{References}

[1] Planck, M. (1910) Acht Vorlesungen ueber Theoretische Physik. S. Hirzel, Leipzig.

[2] Einstein, A. (1917) Physikalische Zeitschrift, 18, 121.

[3] Bohr, N. (1967) On the Quantum Theory of Line Spectra. In: Van der Waerden, B.L., Ed., Sources of Quantum Mechanics, Dover Publications, New York, 95-137.

[4] Bethe, H. (1933) Quantenmechanik der Ein- und Zwei-Elektronenprobleme. In: Geiger, H. and Scheeel, K., Eds., Handbuch der Physik, Vol. 24, Part 1, Springer, Berlin, 273-560.

[5] Condon, E.U. and Shortley, G.H. (1970) The Theory of Atomic Spectra. Cambridge University Press, Cambridge, UK.

[6] Olszewski, S. (2015) Journal of Modern Physics, 6, 1277-1288. http://dx.doi.org/10.4236/jmp.2015.69133

[7] Olszewski, S. (2016) Journal of Modern Physics, 7, 162-174. http://dx.doi.org/10.4236/jmp.2016.71018

[8] Olszewski, S. (2016) Journal of Modern Physics, 7, 827-851. http://dx.doi.org/10.4236/jmp.2016.78076

[9] Olszewski, S. (2016) Journal of Modern Physics, 7, 1004-1020. http://dx.doi.org/10.4236/jmp.2016.79091

[10] Olszewski, S. (2016) Reviews in Theoretical Science, 4, 336-352. http://dx.doi.org/10.1166/rits.2016.1066

[11] Sommerfeld, A. (1931) Atombau und Spektrallinien. 5th Edition, Vol. 1, Vieweg, Braunschweig.

[12] MacDonald, A.H. (1989) Quantum Hall Effect. A Perspective. Kluwer, Milano.

[13] Slater, J.C. (1967) Quantum Theory of Molecules and Solids. Vol. 3, McGraw-Hill, New York.

[14] Lass, H. (1950) Vector and Tensor Analysis. McGraw-Hill, New York.

[15] Matveev, A.N. (1964) Electrodynamics and the Theory of Relativity. Izd. Wyzszaja Szkola, Moscow. (In Russian)

[16] Greiner, W. (1998) Classical Electrodynamics. Springer, New York. http://dx.doi.org/10.1007/978-1-4612-0587-6

[17] Landau, L.D. and Lifshits, E.M. (1969) Mechanics. Electrodynamics. Izd. Nauka, Moscow. (In Russian)

[18] Sommerfeld, A. (1943) Mechanik. Akademische Verlagsgesellschaft, Leipzig.

[19] Born, M. (1933) Optik. Springer, Berlin. http://dx.doi.org/10.1007/978-3-642-99599-6

[20] Van der Waerden, B.L. (1967) Introduction. In: Van der Waerden, B.L., Ed., Sources of Quantum Mechanics, Dover Publications, New York. http://dx.doi.org/10.1007/978-3-662-42424-7 1

[21] Heisenberg, W. (1925) Zeitschrift fuer Physik, 33, 879-893. http://dx.doi.org/10.1007/BF01328377

[22] Olszewski, S. (2014) Journal of Modern Physics, 5, 2022-2029. http://dx.doi.org/10.4236/jmp.2014.518198

[23] Olszewski, S. (2014) Journal of Modern Physics, 5, 2030-2040. http://dx.doi.org/10.4236/jmp.2014.518199

[24] Olszewski, S. (2016) Journal of Modern Physics, 7, 1297-1303. http://dx.doi.org/10.4236/jmp.2016.711114 


\section{Submit or recommend next manuscript to SCIRP and we will provide best service for you:}

Accepting pre-submission inquiries through Email, Facebook, LinkedIn, Twitter, etc.

A wide selection of journals (inclusive of 9 subjects, more than 200 journals)

Providing 24-hour high-quality service

User-friendly online submission system

Fair and swift peer-review system

Efficient typesetting and proofreading procedure

Display of the result of downloads and visits, as well as the number of cited articles

Maximum dissemination of your research work

Submit your manuscript at: http://papersubmission.scirp.org/ 